\title{
Effectiveness of chlorhexidine varnish for preventing caries uncertain
}

\author{
Abstracted from \\ James P, Parnell C, Whelton $\mathbf{H}$. \\ The caries-preventive effect of chlorhexidine varnish in children and adolescents: \\ a systematic review. Caries Res 2010; 44: 333-340. \\ Address for correspondence: Patrice James, Oral Health Services Research Centre, \\ Cork University Dental School and Hospital, Wilton, Cork, Ireland. E-mail: p.james@ucc.ie
}

\section{Questions: In children and adolescents is chlorhexidine varnish effective in preventing caries?}

Data sources Medline, Embase, the Cochrane Central Register of Controlled Trials (CENTRAL), and reference lists of reviews. Study selection Randomised or quasi-randomised controlled trials with blind outcome assessment and a minimum duration of 1 year comparing chlorhexidine varnish to placebo, no treatment or fluoride varnish were included. Split-mouth trials were included but analysed separately. The primary outcome measure was the caries increment determined using the decayed missing and filled surface (DMFS/dmfs) index. Only trials where caries were assessed by visual/visual-tactile or radiographic methods were included.

Data extraction and synthesis Data were extracted by one author and verified by a second. It was planned to present the results graphically in a forest plot and formally assess statistical heterogeneity. However, due to missing data and variation in the reporting of outcomes, this was not possible. Consequently a narrative summary of the results is presented with parallel-group and split-mouth trials considered separately. Heterogeneity was assessed informally.

Results 12 trials were included (eight parallel group, four split-mouth trials) There was considerable variation between trials in the concentration and frequency of application of the chlorhexidine varnish, in baseline caries levels and in background exposure to fluoride. Six parallel group trials reported no statistically significant difference in caries increment in permanent teeth with the application of chlorhexidine varnish compared to placebo or no treatment. The results of 4 split-mouth trials were conflicting: 2 trials found no significant difference in caries increment and 2 reported statistically significant results in favour of chlorhexidine varnish. One trial of the effect of chlorhexidine varnish in primary teeth demonstrated a statistically significant reduction in caries increment. The results of 1 trial comparing chlorhexidine varnish with fluoride varnish for preventing caries in adolescents were equivocal.

Conclusions Evidence regarding the effectiveness of chlorhexidine varnish for preventing caries is inconclusive. Further well-conducted randomised trials are required before chlorhexidine varnish can be recommended for caries prevention.

\section{Commentary}

Caries is one of the most common human diseases. Topical fluoride therapies are often used to prevent caries and have a large and well-reported evidence base. Chlorhexidine varnish could poten- tially be used as it is effective in reducing mutans streptococci (MS) counts in dental plaque as this micro-organism is implicated in caries development.

The introduction described two pre-existing reviews. This review was differentiated from these as it included primary teeth, was restricted to children and carried out an assessment of methodological quality, something that was missing from the previous reviews. However the rationale for the use of chlorhexidine varnish could have been made clearer. There is clear evidence supporting the use of fluoride therapies, it could be argued that there is no need for an alternative.

Search criteria for this review were adequate, though the updated search from 2009-2010 did not include Embase. The inclusion criteria were not ideal as quasi-randomised trials were included. The relationship between selection bias and biased intervention effects is clear. ${ }^{1}$ The authors also included studies with a split-mouth design. These data were analysed separately however it could be argued that these studies should have been excluded due to the risk of a carry-over effect. The authors justified the inclusion of split-mouth designs by quoting Ribiero et al. $^{2}$ who showed that the carry-over effect was negligible for chlorhexidine varnish.

The authors were unable to pool data from different trials as in general the quality of included studies was poor.

The study concluded that evidence for the use of chlorhexidine varnish to prevent caries was inconclusive. The recommendations were that higher quality studies were required, possibly using higher concentrations of chlorhexidine varnish. The authors might also have considered that further studies of chlorhexidine varnish were unwarranted given the availability of fluoride varnish, a therapy with clear evidence of its effectiveness.

\section{Practice points}

- Chlorhexidine varnish does not appear to be effective for caries prevention in children. Well researched interventions such as fluoride varnish remain the therapy of choice for 'in-office' caries prevention.

\section{Paul Ashley}

Paediatric Dentistry, UCL Eastman Dental Institute, London, UK

1. Higgins JPT, Green S (editors). 8.9.1 Rationale for concern about bias. Cochrane Handbook for Systematic Reviews of Interventions Version 5.0.2 [updated September 2009].

2. Ribeiro LG, Hashizume LN, Maltz M. The effect of different formulations of chlorhexidine in reducing levels of mutans streptococci in the oral cavity: a systematic review of the literature. J Dent 2007; 35: 359-370.

Evidence-Based Dentistry (2010) 11, 108. doi:10.1038/sj.ebd.6400754 